
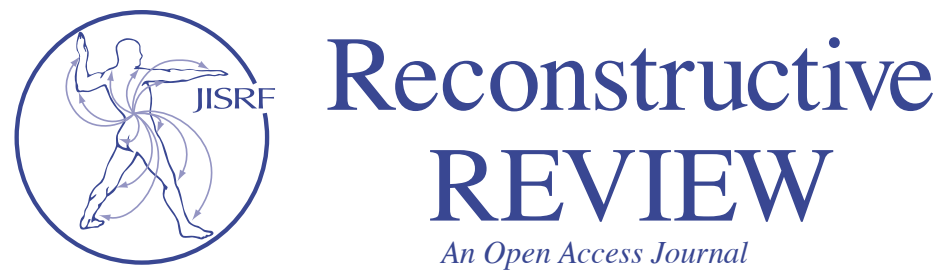

\title{
Range of Motion Comparison Following Total Knee Arthroplasty with and without Patella Resurfacing
}

\author{
Jackels, $M^{1}$; Andrews, $S^{2}$; Matsumoto, $M^{1}$; Mathews, $K^{3}$; Nakasone, $C^{2}$
}

\section{Abstact}

Background: Despite significant evaluation, no consensus has been reach for best clinical practice for resurfacing the patella during total knee arthroplasty. Further complicating the ability to reach a conclusion is the inclusion of several different implant types used in previous research.

Questions/Purpose: The purpose of this study was to compare post-TKA outcomes between two cruciate retaining implants with or without patella resurfacing.

Methods: This retrospective review included 289 patients (380 knees) with a minimum six-month follow-up. All patients received a CR implant, with either a symmetric or an asymmetric tibial baseplate. Post-TKA knee flexion was categorized as $<120^{\circ}$ and $\geq 120^{\circ}$ and knee extension classified as $0^{\circ}$ or $>0^{\circ}$ and required knee manipulations were noted. Descriptive, nonparametric statistics were performed and a multivariate logistic regression was performed to determine risk of poor range of motion and manipulations.

Results: Age was significantly lower in the resurfaced group ( $\mathrm{p}=0.001)$ and the resurfaced group had longer tourniquet time $(\mathrm{p}=0.003)$. The symmetric-resurfaced group had $\geq 120^{\circ}$ of flexion and full extension in $72 \%$ and $98.7 \%$ of patients, respectively. Compared to symmetric-resurfaced, all other groups had a significantly greater risk of not reaching $120^{\circ}$ of knee flexion $(\mathrm{p}<0.05)$. There were no significant differences in the risk of requiring a MUA between groups $(\mathrm{p}>0.06)$.
Conclusions: The effect of resurfacing the patella on post-TKA outcomes may be influenced by tibial implant design. Compared to all other combinations, a symmetric tibial baseplate and resurfaced patella resulted in the highest percentage of patients reaching $\geq 120^{\circ}$, with a low incidence of manipulations.

\section{Background}

The reported rate of patellar resurfacing during primary total knee arthroplasty (TKA) has ranged from 3\% in Sweden to $98 \%$ in the United States, remaining essentially unchanged over the last decade [1]. This large range highlights the variation of surgeon opinion and decades of research has provided little to no conclusive evidence for best standard of practice for patella resurfacing. Evidence against routine patella resurfacing primarily includes cadaveric and anatomic studies, reporting abnormal kinematics, contact areas and loading characteristics [2-ㅛ $]$. Conversely, several meta-analyses conclude a lower incidence of anterior knee pain and fewer revisions following routine patella resurfacing but fail to report consistent clinically meaningful differences [2-14].

Previous joint registry evaluations have alluded to the influence of implant design on outcomes following TKA

Keywords: range of motion; total knee arthroplasty; patella resurfacing

Level of Evidence: III 
with or without patella resurfacing [15-18]. The lack of consensus is likely due to the myriad of implant designs available and from which research has been conducted, which include fundamental differences in function and stability. However, previous research has suggested that cruciate retaining $(\mathrm{CR})$ implants with patella resurfacing may result in a lower incidence of anterior knee pain and fewer revisions when compared to posterior stabilized implants $[\underline{12}, \underline{13}, \underline{19}]$. Unfortunately, even within CR implant research, multiple implant designs are present and broad application is limited.

A primary design difference in CR implants is the presence of either a symmetric or an asymmetric tibial baseplate, with modern designs commonly implanted with an ultra-congruent (UC) tibial insert. Despite the wide use and high success of these implants $[\underline{20}, \underline{21}]$, the influence of patella resurfacing and subsequent clinical outcomes, specifically post-operative knee range of motion (ROM), remain uncertain. Therefore, the purpose of this study was to compare post-TKA knee range of motion for patients with a minimum six month follow up between CR implants with symmetric and asymmetric tibial baseplates, with or without patella resurfacing.

\section{Materials and Methods}

This institutional review board approved, retrospective review included a consecutive cohort of 289 patients (380 knees) having undergone primary TKA for the treatment of osteoarthritis between January 2016 and January 2019 and had a minimum six month post-operative clinical assessment. All procedures were performed by a single, high volume fellowship trained orthopedic surgeon at a community hospital with nearly 10 years of fast track arthroplasty type delivery of care. The standard of care for primary TKA included the use of a tourniquet and a medial parapatellar approach with eversion of the patella and removal of the patellar fat pad for visualization. The posterior cruciate ligament was sacrificed in all procedures. Balancing of the knee was performed utilizing standard spacer blocks and alignment rods to assess intraoperative alignment and extension and flexion gap balance. Medial or lateral soft tissue releases were performed if necessary to obtain balance. Reduction of bony osteophytes were also performed where necessary to achieve proper knee balance. Extension and flexion gap balancing was performed in an identical manner for both systems as both systems are nearly identical in design regarding the femoral components. Patellar tracking was tested with extension and flexion of the knee without pressure on the patellae. The study surgeon routinely uses two implant types to perform TKA. Both systems have nearly identical femoral designs utilizing a multi-radius femoral component with similar trochlear designs. The major difference between the two systems are largely related to the design of the tibial baseplate. Therefore, over the study period, the two CR implants were used without preference for patient demographics or disease progression. Although not part of a formal randomization process, patients were randomly assigned by the surgeon without regard for patient demographics, severity of arthritis or deformity present to receive either a symmetric tibial baseplate (Balanced Knee® System, Ortho Development Corporation, Draper, UT) or an asymmetric tibial baseplate (Persona ${ }^{\circledR}$, Zimmer Biomet, Warsaw, IN). Both systems were used with the corresponding UC tibial insert.

Until September 2017, the senior surgeon would have been classified as "usually" resurfacing the patella, with over $90 \%$ of patients undergoing patella resurfacing [17]. Due to lack of definitive data to support or condemn either practice regarding the patellae, the senior surgeon switched to a "rarely" patella resurfacing classification following September 2017, during which time less than 10\% of patients underwent patella resurfacing [17]. During this time, patella resurfacing was rarely performed regardless of the condition of the native patellae. In all cases of patella resurfacing, pre- and post-patella resurfacing measurements were taken using a caliper to measure the thickest portion of the patella prior to resection of the articular surface and then following completion of cemented fixation of an all polyethylene symmetric dome shaped patella.

Following TKA, immediate and unrestricted, full weight bearing was allowed as tolerated, with no knee ROM restrictions. Physical therapy evaluation and treatment began on the day of surgery and continued twice daily until cleared for discharge. All patients received outpatient rehabilitation beginning within a week of hospital discharge and continued for six weeks. Standard physical therapy ROM goals were to equal or exceed preoperative ROM, with a minimum goal of $120^{\circ}$ of flexion targeted if possible. Patients with (1) less than $90^{\circ}$ of flexion, (2) greater than $10^{\circ}$ of a flexion contracture and/or (3) greater than $30^{\circ}$ loss of total motion from preoperative ROM measurements at the six week post-TKA clinic visit were considered candidates for manipulation under anesthesia (MUA). Immediately following MUA, physical therapy was prescribed for five consecutive days, followed by three times per week until therapy goals were met or further progress in ROM could not be achieved.

Patient demographics were collected for each patient at the time of surgery, including age, body mass index (BMI) and American Society of Anesthesiologists' (ASA) clas- 
sification determined by the core group of anesthesiologists experienced in arthroplasty care. Pre- and post-operative knee flexion and extension were recorded by the senior surgeon or physician assistant. The decision for MUA was typically made between 6 weeks and three months following TKA, therefore, would have been noted prior to the six month clinic visit.

Data were analyzed across four groups, designated by tibia component design and patella resurfacing status, including asymmetric-resurfaced, asymmetric-non-resurfaced, symmetric-resurfaced and symmetric-non-resurfaced. Range of motion was evaluated both as a continuous variable and as a categorical variable, including knee flexion categorized as less than $120^{\circ}$ and equal to or greater than $120^{\circ}$ and

Table 1. Patient Characteristics and Perioperative Variables by Group

\begin{tabular}{|c|l|l|l|l|l|}
\hline \multicolumn{1}{|c|}{ Patella } & \multicolumn{2}{|c|}{ Resurfaced } & \multicolumn{2}{c|}{ Non-Resurfaced } & \\
\hline Tibia & $\begin{array}{c}\text { Asymmetrical } \\
(\mathbf{N = 5 3 )}\end{array}$ & $\begin{array}{c}\text { Symmetrical } \\
(\mathbf{N = 1 2 5})\end{array}$ & $\begin{array}{c}\text { Asymmetrical } \\
(\mathbf{N = 1 2 3})\end{array}$ & $\begin{array}{c}\text { Symmetrical } \\
(\mathbf{N = 8 0})\end{array}$ & p-value \\
\hline Age (years)* & $67.5(8.2)$ & $68.1(8.4)$ & $71.4(7.7)$ & $71.4(9.5)$ & 0.001 \\
\hline BMI (kg/m2)* & $30.8(6.1)$ & $30.7(5.8)$ & $28.8(5.6)$ & $29.7(5.8)$ & 0.099 \\
\hline Gender* & & & & & \\
\hline Male & $10(25.0 \%)$ & $43(43.9 \%)$ & $41(43.6 \%)$ & $22(38.6 \%)$ & 0.178 \\
\hline Female & $30(75.0 \%)$ & $55(56.1 \%)$ & $53(56.4 \%)$ & $35(61.4 \%)$ & \\
\hline Procedure & & & & & 0.252 \\
\hline Unilateral & $27(50.9 \%)$ & $71(56.8 \%)$ & $65(52.8 \%)$ & $34(42.5 \%)$ & \\
\hline Bilateral* & $13(29.1 \%)$ & $27(43.2 \%)$ & $29(47.2 \%)$ & $23(57.5 \%)$ & \\
\hline $\begin{array}{c}\text { Tourniquet } \\
\text { Time }\end{array}$ & $40.5(8.6)$ & $40.2(10.8)$ & $37.7(9.6)$ & $37.4(10.9)$ & 0.003 \\
\hline Surgical Time & & & & & \\
\hline Unilateral & $66.1(12.0)$ & $71.8(21.5)$ & $63.6(12.6)$ & $65.9(16.8)$ & 0.048 \\
\hline Bilateral* & $172.5(18.9)$ & $167.0(20.6)$ & $153.9(15.5)$ & $154.2(18.9)$ & 0.005 \\
\hline
\end{tabular}

$*$ = bilaterals included once, therefore, sample size is number of patients

Presented at Mean (standard deviation) or Frequency (\% of total)

$\mathrm{N}=$ number of knees

knee extension classified as $0^{\circ}$ or

greater than $0^{\circ}$. Categorical classification of range of motion was based on standard post-operative range of motion targets, in which $120^{\circ}$ of knee flexion and $0^{\circ}$ of knee extension are established patient goals. Post-operative ROM data were taken from the last clinic visit attended, with a minimum of six month follow up required. Group differences in categorical data were evaluated by Chi Square tests. Continuous data were non-parametric as determined by the Kolmogorov-Smirnov test; therefore, Kruskal-Wallis tests were performed to determine group differences. Multiple Mann-Whitney Tests were performed to determine differences between asymmetric and symmetric for resurfaced and non-resurfaced patella. A multivariate logistic regression was performed for post-operative ROM and MUA, with symmetric tibial and resurface patella as the reference category for all analyses. Results were presented at odds ratios (OR) and 95\% confidence intervals (CI). All statistical analyzes were performed using SPSS vs 25 with a significance level of $\mathrm{p}<0.05$.

\section{Results}

Overall, the average age of all patients was $69.73(8.5)$ years old and average BMI was 29.88 (5.8). Group descriptive are presented in Table 1, with significant main effects noted in age $(p=0.001)$, tourniquet time $(p=0.003)$ and surgical time for both unilateral $(\mathrm{p}=0.048)$ and bilateral $(\mathrm{p}=0.005)$ procedures. There was no significant difference between tibial baseplate groups for resurfaced and non-resurfaced patella (Mann-Whitney).

No significant group differences were present in preoperative ROM (Table 2). Group differences were present for all post-operative ROM variables, including knee flexion ( $\mathrm{p}<0.001$ ) and knee extension ( $\mathrm{p}=0.003)$, with symmetric-resurfaced having the greatest flexion and extension. Additionally, knee flexion was significantly greater in symmetric-resurfaced compared to asymmetric-resurfaced $(p<0.001)$. Knee extension was significantly less in symmetric-non-resurfaced compared to asymmetric-nonresurfaced $(\mathrm{p}=0.010)$. Categorically, ROM goals were most commonly met with symmetric-resurfaced for flexion $(72 \%)$ and symmetric-non-resurfaced for extension (98.7\%). Range of motion goals were least commonly met with symmetric-non-resurfaced for flexion $(51.2 \%)$ and asymmetric-non-resurfaced for extension $(89.4 \%)$. There was not significant group difference for MUAs, however, asymmetric-non-resurfaced had the greatest occurrence, with $8.9 \%$ of patients requiring MUA.

Compared to symmetric-resurfaced, all other groups had a significantly greater risk of not reaching $120^{\circ}$ of knee flexion $(\mathrm{p}<0.05)$ (Table 3). Additionally, asymmetricnon-resurfaced had a significantly greater risk of not reaching full extension compared to the symmetric-resurfaced 
Table 2. Functional Outcomes by Group - Mean(SD)/Frequency/(\%)

\begin{tabular}{|l|l|l|l|l|l|}
\hline \multicolumn{1}{|c|}{ Patella } & \multicolumn{2}{c|}{ Resurfaced } & \multicolumn{2}{c|}{ Non-Resurfaced } & \\
\hline \multicolumn{1}{|c|}{ Tibia } & \multicolumn{1}{c|}{$\begin{array}{c}\text { Asymmetrical } \\
(\mathbf{N = 5 3 )}\end{array}$} & $\begin{array}{c}\text { Symmetrical } \\
(\mathbf{N = 1 2 5})\end{array}$ & $\begin{array}{c}\text { Asymmetrical } \\
(\mathbf{N = 1 2 3 )}\end{array}$ & $\begin{array}{c}\text { Symmetrical } \\
(\mathbf{N = 8 0})\end{array}$ & p-value \\
\hline Pre-Flex & $114.5(14.2)$ & $117.9(14.2)$ & $115.4(14.9)$ & $114.9(14.6)$ & 0.196 \\
\hline Pre-Ext & $3.8(5.5)$ & $3.9(7.5)$ & $4.2(6.9)$ & $4.0(5.3)$ & 0.802 \\
\hline Post-Flex & $113.3(10.9)$ & $119.8(10.2)^{*}$ & $115.5(10.2)$ & $114.1(12.5)$ & $<0.001$ \\
\hline Post-Ext & $0.6(2.9)$ & $0.2(1.8)$ & $1.2(4.0)$ & $0.1(0.6)^{\wedge}$ & 0.003 \\
\hline PostFlex<120 & $23(43.4 \%)$ & $35(28.0 \%)$ & $50(40.7 \%)$ & $39(48.8 \%)$ & 0.017 \\
\hline PostExt>0 & $3(5.7 \%)$ & $2(1.6 \%)$ & $13(10.6 \%)$ & $1(1.3 \%)$ & 0.004 \\
\hline MUA & $1(1.9 \%)$ & $4(3.2 \%)$ & $11(8.9 \%)$ & $2(2.5 \%)$ & 0.062 \\
\hline
\end{tabular}

Pre = preoperative $;$ Post $=$ postoperative Flex $=$ knee flexion $;$ Ext $=$ knee extension

$\mathrm{N}=$ number of knees; MUA = manipulation under anesthesia; $\mathrm{SD}=$ standard deviation

$*$ = significantly greater than asymmetrical-resurfaced $(\mathrm{p}<0.001)$ (Mann-Whitney)

$\wedge=$ significantly less than asymmetrical-non-resurfaced ( $\mathrm{p}=0.010)$ (Mann-Whitney)

Table 3. Odds for Obtaining Post-Operative Functional Goal

\begin{tabular}{|c|c|c|c|c|}
\hline & Tibia & Patella & OR $(95 \% \mathrm{CI})$ & p-value \\
\hline \multirow[t]{4}{*}{ Post-Flexion* } & Symmetrical & Resurfaced & Reference & \\
\hline & Symmetrical & Non-Resurfaced & $0.409(0.227-0.735)$ & 0.003 \\
\hline & Asymmetrical & Resurfaced & $0.507(0.260-0.990)$ & 0.047 \\
\hline & Asymmetrical & Non-Resurfaced & $0.568(0.334-0.966)$ & 0.037 \\
\hline \multirow[t]{4}{*}{ Post-Extension^ $^{\wedge}$} & Symmetrical & Resurfaced & Reference & \\
\hline & Symmetrical & Non-Resurfaced & $1.285(0.115-14.403)$ & 0.839 \\
\hline & Asymmetrical & Resurfaced & $0.271(0.044-1.671)$ & 0.160 \\
\hline & Asymmetrical & Non-Resurfaced & $0.138(0.030-0.623)$ & 0.010 \\
\hline \multirow[t]{4}{*}{ MUA } & Symmetrical & Resurfaced & Reference & \\
\hline & Symmetrical & Non-Resurfaced & $1.289(0.231-7.208)$ & 0.772 \\
\hline & Asymmetrical & Resurfaced & $1.719(0.188-15.753)$ & 0.632 \\
\hline & Asymmetrical & Non-Resurfaced & $0.337(0.104-1.088)$ & 0.069 \\
\hline
\end{tabular}

$\mathrm{OR}=$ odds ration $; \mathrm{CI}=$ confidence interval; $\mathrm{MUA}=$ manipulation under anesthesia

$*$ = functional goal was above $120^{\circ}$ of knee flexion by six months

$\wedge=$ functional goal was above $0^{\circ}$ of knee extension by six months

** OR below 1 indicates increased risk for poor outcome compared to reference the current study aimed to evaluate the influence of patella resurfacing on post-operative ROM outcomes, using two distinctly different CR implants with an UC insert. The results of the current study indicate that resurfacing the patella when using a symmetric tibial implant provided the best post-operative knee flexion outcomes, with $72 \%$ of patients successfully achieving the established clinical goal of greater than $120^{\circ}$ of knee flexion. Although this combination resulted in the second best knee extension outcomes, only two patients $(1.6 \%)$ did not achieve full extension and only four patients (3.2\%) required MUA.

Previous research and meta-analyses commonly use post-operative ROM as the primary outcome in the evaluation of patella resurfacing [913]. The results of those studies concluded that a resurfaced patella significantly increased post-operative ROM, particularly knee flexion. Interestingly, the results of the current study clearly indicate that post-operative knee flexion in patients with a resurfaced patella was dependent on the type of tibial implant used. Specifically, symmetric-resurfaced patients had the greatest knee flexion (average $119.8^{\circ}$ ) while asymmetricresurfaced patients had the lowest knee flexion (average $113.3^{\circ}$ ). Although knee flexion was not significantly different in the non-resurfaced group between tibial implants, failure to achieve full extension follow- group. There were no significant differences in the risk of requiring a MUA between groups.

\section{Discussion}

Previous research has failed to provide adequate evidence to recommend for or against resurfacing the patella during TKA. The use of several different implant designs has further limited the ability for previous research to isolate the influence of patella resurfacing. Therefore, ing TKA was significantly greater in the asymmetric-nonresurfaced group. The lack of full knee extension in this group led to $10.6 \%$ of patients not meeting their post-operative ROM goals and $8.9 \%$ of those patients required a MUA, which was the most of all groups.

Significant differences in post-TKA ROM were noted between the four groups and, in the context of clinical goals of $120^{\circ}$ of knee flexion and $0^{\circ}$ of knee extension, these small differences in ROM are likely clinically relevant. Compared to symmetric-resurfaced, the risk for not achieving $120^{\circ}$ of knee flexion was significantly greater 
for all other groups. More specifically, the combination of a symmetric tibial baseplate with a resurfaced patella provided the best environment for a patient to obtain $120^{\circ}$ of knee flexion. This distinction was not as clear for extension, as only the asymmetric-non-resurfaced patients had a significantly greater risk for not achieving full knee extension compared to symmetric-resurfaced. These results, coupled with the low incidence of MUA, suggests that when using a CR implant and UC insert during TKA, the use of a symmetric tibial baseplate and resurfacing the patella may provide the best post-operative range of motion outcomes.

As expected, resurfacing the patella significantly increased both tourniquet and surgical time for both unilateral and bilateral TKAs. Unfortunately, the increase in tourniquet and surgical time was accompanied by an increasing patient BMI, with positive correlations between these variables previously described in literature [22]. Nonetheless, the difference in tourniquet time of less than three minutes was clinically insignificant. Even with the compounding tourniquet time for bilateral patients, surgical time increased an average of only 16 minutes.

There are a few limitations regarding this study. This was a retrospective chart review evaluating only post-operative range of motion outcomes. The lack of formal randomization for this study could have introduced a selection bias when resurfacing the patella. However, the lack of group differences in pre-operative ROM adds confidence to our results. Secondly, only ROM outcomes were collected during patient clinic visits with no patient reported outcomes to supplement these results. Furthermore, the condition of the patella was not graded at the time of surgery; therefore, no comment can be made regarding the relationship between severity of patellofemoral arthritis at the time of surgery, patella treatment and post-operative ROM. Lastly, while the femoral components used in the current study are nearly identical in design, there are very subtle design differences that could have had an impact on the results presented. However, it is the similarity of the femoral designs of the two systems studied here used with significantly different baseplate designs that may strengthen these findings as the tibial baseplate design was the only major difference between the systems. Ongoing, prospective research at the current institution includes a randomization of patients and collection of both patella grading and patient reported outcomes.

\section{Conclusion}

Based on the results of the current study, the effect of resurfacing the patella on postoperative ROM may be influenced by tibial implant design. In the current study, the combination of a symmetric tibial baseplate and a resurfaced patella provided the best overall post-operative ROM, with a low incidence of MUAs. Additionally, all other surgical combinations provided either a similar risk or an increased risk for not achieving full ROM. These preliminary results should be further evaluated before a definitive recommendation can be made for patella resurfacing.

\section{References}

1. Fraser JF and Spangehl MJ. International Rates of Patellar Resurfacing in Primary Total Knee Arthroplasty, 2004-2014. J Arthroplasty 32(1): 83-86, 2017.

2. Fuchs S, Schutte G, Witte H and Rosenbaum D. Retropatellar contact characteristics in total knee arthroplasty with and without patellar resurfacing. Int Orthop 24(4): 191-193, 2000.

3. Barink M, Meijerink H, Verdonschot N, van Kampen A and de Waal Malefijt M. Asymmetrical total knee arthroplasty does not improve patella tracking: a study without patella resurfacing. Knee Surg Sports Traumatol Arthrosc 15(2): 184-191, 2007.

4. Wurm S, Kainz H, Reng W and Augat P. The influence of patellar resurfacing on patellar kinetics and retropatellar contact characteristics. J Orthop Sci 18(1): 6169, 2013.

5. Slevin O, Schmid FA, Schiapparelli FF, Rasch H, Amsler F and Hirschmann MT. Coronal femoral TKA position significantly influences in vivo patellar loading in unresurfaced patellae after primary total knee arthroplasty. Knee Surg Sports Traumatol Arthrosc 25(11): 3605-3610, 2017.

6. Matsuda S, Ishinishi T, White SE and Whiteside LA. Patellofemoral joint after total knee arthroplasty. Effect on contact area and contact stress. J Arthroplasty 12(7): 790-797, 1997.

7. Benjamin JB, Szivek JA, Hammond AS, Kubchandhani Z, Matthews AI, Jr. and Anderson P. Contact areas and pressures between native patellas and prosthetic femoral components. J Arthroplasty 13(6): 693-698, 1998.

8. Singerman R, Gabriel SM, Maheshwer CB and Kennedy JW. Patellar contact forces with and without patellar resurfacing in total knee arthroplasty. J Arthroplasty 14(5): 603-609, 1999.

9. Chen K, Li G, Fu D, Yuan C, Zhang Q and Cai Z. Patellar resurfacing versus nonresurfacing in total knee arthroplasty: a meta-analysis of randomised controlled trials. Int Orthop 37(6): 1075-1083, 2013.

10. Migliorini F, Eschweiler J, Niewiera M, El Mansy Y, Tingart M and Rath B. Better outcomes with patellar resurfacing during primary total knee arthroplasty: a metaanalysis study. Arch Orthop Trauma Surg 139(10): 1445-1454, 2019.

11. Grassi A, Compagnoni R, Ferrua P, Zaffagnini S, Berruto M, Samuelsson K, Svantesson E and Randelli P. Patellar resurfacing versus patellar retention in primary total knee arthroplasty: a systematic review of overlapping meta-analyses. Knee Surg Sports Traumatol Arthrosc 26(11): 3206-3218, 2018.

12. Nizard RS, Biau D, Porcher R, Ravaud P, Bizot P, Hannouche D and Sedel L. A meta-analysis of patellar replacement in total knee arthroplasty. Clin Orthop Relat Res (432): 196-203, 2005.

13. Parvizi J, Rapuri VR, Saleh KJ, Kuskowski MA, Sharkey PF and Mont MA. Failure to resurface the patella during total knee arthroplasty may result in more knee pain and secondary surgery. Clin Orthop Relat Res 438: 191-196, 2005.

14. Teel AJ, Esposito JG, Lanting BA, Howard JL and Schemitsch EH. Patellar Resurfacing in Primary Total Knee Arthroplasty: A Meta-Analysis of Randomized Controlled Trials. J Arthroplasty 34(12): 3124-3132, 2019.

15. Lygre SH, Espehaug B, Havelin LI, Vollset SE and Furnes O. Does patella resurfacing really matter? Pain and function in 972 patients after primary total knee arthroplasty. Acta Orthop 81(1): 99-107, 2010.

16. Maney AJ, Frampton CM and Young SW. Age and Prosthetic Design as Risk Factors for Secondary Patella Resurfacing. J Arthroplasty 35(6): 1563-1568, 2020.

17. Maney AJ, Koh CK, Frampton CM and Young SW. Usually, Selectively, or Rarely Resurfacing the Patella During Primary Total Knee Arthroplasty: Determining the Best Strategy. J Bone Joint Surg Am 101(5): 412-420, 2019. 
18. Coory JA, Tan KG, Whitehouse SL, Hatton A, Graves SE and Crawford RW. The Outcome of Total Knee Arthroplasty With and Without Patellar Resurfacing up to 17 Years: A Report From the Australian Orthopaedic Association National Joint Replacement Registry. J Arthroplasty 35(1): 132-138, 2020.

19. van der Merwe JM and Mastel MS. Controversial Topics in Total Knee Arthroplasty: A 5-Year Update (Part 1). J Am Acad Orthop Surg Glob Res Rev 4(1): e1900047, 2020

20. Galea VP, Rojanasopondist P, Connelly JW, Bragdon CR, Huddleston JI, 3rd, Ingelsrud LH, Malchau H and Troelsen A. Changes in Patient Satisfaction Following Total Joint Arthroplasty. J Arthroplasty 35(1): 32-38, 2020.

21. Singh AD. Retrospective study of asymmetric vs symmetric tibial plates and ultracongruent vs posterior stabilized inserts in Indian population: An Indian experience of Natural Knee II. J Clin Orthop Trauma 7(Suppl 2): 184-190, 2016.

22. Harato K, Yagi M, Fujita N, Kobayashi S, Ohya A, Kaneda K, Iwama Y, Nakamura $\mathrm{M}$ and Matsumoto $\mathrm{M}$. Effect of body mass index on surgical times of lumbar laminoplasty and lower limb arthroplasties. BMC Musculoskelet Disord 20(1) $416,2019$.
SUBMISSION HISTORY

Submitted: June 30, 2020

Reviewed: September 6, 2021

Revised October 8, 2021

Accepted: October 8, 2021

Published: October 14, 2021

\section{AUTHOR AFFILIATIONS}

1 Melissa K. Jackels, MS; Maya Y. Matsumoto, BA John A. Burns School of Medicine University of Hawai'i at Manoa, 651 llalo Street Honolulu, $\mathrm{HI}$

2 Samantha N. Andrews, PhD; Cass k. Nakasone, MD

(1) Straub Medical Center, 888 S. King St., Honolulu, HI (2) John A. Burns School of Medicine, University of Hawai'i, Manoa, 651 Ilalo Street Honolulu, HI Department of Surgery

3 Kristin Mathews, MS Straub Medical Center, 888 S. King St., Honolulu, HI

(Direct inquires to Samantha Andrews, samantha.andrews@straub.net)

\section{AUTHOR DISCLOSURES}

- The authors declare that there is no conflict of interest in connection with this submitted article.

\section{COPYRIGHT \& OPEN ACCESS}

(C) 2021 Jackels, Andrews, Matsumoto, Nakasone, Mathews. All rights reserved Authors retain copyright and grant the journal right of first publication with the work. Reconstructive Review is an open access publication and follows the Creative Commons Attribution-NonCommercial CC BY-NC. This license allows anyone to download works, build upon the material, and share them with others for non-commercial purposes as long as they credit the senior author, Reconstructive Review, and the Joint Implant Surgery \& Research Foundation (JISRF). An example credit would be: "Courtesy of (senior author's name), Reconstructive Review, JISRF, Chagrin Falls, Ohio". 